\title{
La Red Colombiana de Hipertensión Pulmonar: elementos de gestión y gobernanza científica
}

\author{
Mauricio Orozco-Levi, MD, PhD, MSc. ${ }^{1}$ \\ Rafael Conde Camacho, MD, MSc. ${ }^{1}$ \\ Manuel Conrado Pacheco, MD, MSc. ${ }^{1}$ \\ Alejandro Londoño Villegas, MD. ${ }^{1}$
}

Lista de miembros de la Red Colombiana de Hipertensión Pulmonar

Mauricio Orozco Levi, MD, PhD, MSc; Alejandro Londoño Villegas, MD; Héctor Ortega, MD; Rafael Enrique Conde Camacho, MD; Liliana Fernández Trujillo, MD; Manuela Tobón Trujillo, MD; Natalia Uribe Giraldo, MD; Jorge E. Ortega, MD; Gustavo Gómez Correa, MD; Beatriz Cadavid Rodríguez, MD; Juan Fernando Carvajal Estupiñán, MD; Luis Ernesto Téllez Mosquera, MD; Leslie Katherine Vargas Ramírez, MD; Manuel Conrado Pacheco, MD, MSc; Donaldo Díaz Murillo, MD; John Alexander Ramírez Martínez, MD; Solón Navarrete Hurtado, MD; José Gabriel Espinoza Espinoza, MD; Héctor Augusto Escalante Mora, MD; Alejandro Villamil Munévar, MD; Ingrid Johana Martínez R., MD; Eneida Martina Fonseca Ortiz, MD; Fabio Bolívar Grimaldos, MD; Carlos Aguilar, MD; José Coppiano, MD; José Alfredo Posada, MD; Jaime Echeverry, MD; Jaime Sánchez, MD; Ángela Giraldo, MD; Martin Romero, MD; Lina Saldarriaga, MD; Juan M. Cárdenas, MD; Mauricio Céspedes, MD; José Luis Blanco, MD; Carlos Jaime Velásquez, MD; Ricardo Gómez Palau, MD; Giovanny Osorno, MD; Bernardo Muñoz Palacio, MD; Claudia Patricia Díaz Bossa, MD; Felipe Campo Campo, MD; Julio Cesar Forero Forero, MD; Luz Adriana Ocampo Ocampo, MD; Margarita María Zapata Sánchez, MD; Juan Esteban Gómez, MD; Julián Cortes, MD; Diana Tiga Loza, ENF, PhD, Juliana De Luque Figueroa, ENF, Alba Ramírez-Sarmiento, FT, PhD. Subvenciones: HAPredco (Red Colombiana de Hipertensión Pulmonar) ha contado en 2019, 2020 y 2021 con apoyos irrestrictos de BAYER, JANSSEN y ABBOT'T para acciones específicas definidas entre las partes y con contabilidad central en la Asociación Colombiana de Neumología y Cirugía del Tórax (ASONEUMOCITO) y la Sociedad Colombiana de Cardiología.
${ }^{1}$ En nombre de la Red Colombiana de Hipertensión Pulmonar.

\section{Autor de Correspondencia Mauricio Orozco-Levi Correo electrónico: mauricioorozcolevi@gmail.com}

Recibido: 8/05/ 2021

Aceptado: 10/06/2021 


\section{Constitución de la Red Colombiana de Hipertensión Pulmonar, "HAPredco" mediante diseño de precisión}

El presente documento resume el marco de colaboración denominado "Red Colombiana de Hipertensión Pulmonar" entre miembros de diversas instituciones y especialidades médicas y paramédicas, que tienen intereses académicos y científicos en el diagnóstico, tratamiento y/o investigación en áreas diversas de la Hipertensión Pulmonar (HP). Esta iniciativa denominada con el acrónimo "HAPredco", se justifica ampliamente por la relevancia científica de viabilizar un intercambio gremial, transdisciplinar y continuo mediante una Plataforma de Acción Continuada (PAC) de gestión de la información, experiencias, bases de datos y experticias alrededor de la hipertensión pulmonar $(1,2)$.

En particular, la HAPredco se ha concebido como una estructura escalonada (no necesariamente secuencial) en donde el resultado final estará representado por la participación de miembros de especialidades médicas y profesiones afines de toda Colombia y, eventualmente, escalable a otros países de Latinoamérica. El Primer Escalón de la Red estuvo constituido por la declaración de intenciones entre profesionales de centros hospitalarios de Bogotá, Bucaramanga, Cali y Medellín, y han ido incluyendo continuamente profesionales de otras ciudades. Hemos adelantado los lineamientos generales aquí definidos como "Propuesta Marco para la Constitución de la Red Colombiana de Hipertensión Pulmonar". La consolidación de la iniciativa y este documento de publicación representa el Segundo Escalón de crecimiento de la HAPredco. Este nuevo paso está dirigido a informar, hacer partícipe y solicitar aval como un proyecto original integrado a ASONEUMOCITO y otras agremiaciones profesionales nacionales. Todo lo anterior ha permitido acelerar hacia el Tercer Escalón, representado por la base de datos transinstitucional en un entorno aplicativo basado en la Web y con accesos ilimitados para los diversos investigadores y colaboradores con credenciales participativas. El número de escalones subsiguientes, estará determinado por el número de estrategias diferentes que haya que implementar, según lo demuestre la gestión gremial. Este "diseño de precisión” permitirá ofertar y facilitar el proyecto a los demás países latinoamericanos, adoptándolo o adaptándolo según proceda, a las particularidades de cada país y la actividad de los profesionales interesados (3).

\section{¿Qué es y qué no es la HAPredco?}

Por definición, la "Red" busca crear una plataforma gremial, transinstitucional y de acción continua que permita albergar los intereses consensuados de múltiples participantes latinoamericanos, bajo la única premisa de ofrecer elementos útiles y potenciales beneficios a los pacientes con hipertensión pulmonar que residan en Colombia, siendo un proyecto escalable a otros países (4). Creemos que este modelo de participación gremial puede sentar bases y definir estrategias para potenciar los objetivos específicos de ASONEUMOCITO y otras sociedades científicas alrededor de la hipertensión pulmonar. Por una parte, la HAPredco tiene características particulares de alto valor por su visión, misión, objetivos, y características (apertura, modelo de gestión y gobernanza) que, en conjunto, justifican una declaración de intenciones sólida, relevante y pertinente mediante esta propuesta. Nuestra iniciativa promueve que la Red represente una oferta particular de interacción gremial inicialmente centrada en Colombia, con proyección ampliable al resto de Latinoamérica. Esta fase inicial permitirá desarrollar instrumentos de interacción suficientemente afinados para informar, invitar y difundir la iniciativa de la Red a otros países latinoamericanos, según su propio interés de pertenencia a la misma y a otros grupos referentes externos, idealmente bajo la interacción normativa de ALAT (Asociación Latinoamericana del Tórax). Además, permitirá avanzar hacia un modelo de interacción ampliable a otras sociedades, organizaciones y países de Latinoamérica interesados en todo lo relacionado a los pacientes con hipertensión pulmonar. Por otra parte, la HAPredco no tiene características excluyentes, no tiene representación de un país único, no es un proyecto de investigación único o específico, no depende y no está limitada a una institución, organización, empresa o sociedad única. No es 
una estrategia de tiempo definido, no tiene una orientación disciplinaria única, y no depende de un investigador, gestor o grupo únicos (5).

\section{Misión de HAPredco}

Integrar a la HAPredco a todo individuo, sociedades y organizaciones de Colombia interesados en la Hipertensión Pulmonar, bajo un marco de gobernanza propio.

\section{Visión de HAPredco}

Integrar a través de objetivos comunes de investigación y lo relacionado a pacientes con hipertensión pulmonar, la HAPredco, y sociedades científicas internacionales, de tal forma que en cinco años sea una organización académica sólida y multifacética que desarrolle actividades de interés científico alrededor de la enfermedad.

\section{Objetivos de la HAPredco}

1. Caracterizar la problemática de la HP en Colombia.

2. Resolver los interrogantes epidemiológicos, clínicos y económicos.

3. Desarrollar investigaciones en población propia y compartida.

4. Normalizar el conocimiento relacionado con el diagnóstico, tratamiento y seguimiento de la HP en la región.

5. Promover la consecución de fondos para actividades científicas y académicas propias.

6. Ofrecer asesoría en problemas particulares de pacientes con HP en Colombia, cada uno en su respectivo centro y región.

\section{Características de la HAPredco}

1. La Red es una plataforma con orientación fundamentalmente académica y científica.

2. La Red es original, pertinente y relevante, por abarcar aspectos sociales, clínicos, económicos y académicos de la hipertensión pulmonar, con base inicial en Colombia y México, y con extensión escalonada a otros países de Latinoamérica.

3. La Red es incluyente y promueve el carácter transdisciplinar y multiinstitucional, con una visión de futuro extendida a toda Latinoamérica.

4. La Red es de tipo transdisciplinar, con participación de múltiples especialidades y disciplinas aliadas.

5. La Red es factible, pues se cuenta con la intención de suficientes profesionales de la salud, instituciones y sociedades interesadas en el tema de los pacientes con hipertensión pulmonar.

6. Los alcances de la Red tienen que ver con la voluntad, actitud, competencias y disposición del personal de la salud y asociados.

7. La ejecución del trabajo en Red es práctica, pues propende hacia la aplicación de los conocimientos y estándares de diagnóstico y manejo de la hipertensión pulmonar en Colombia y México, así como del resto de países de Latinoamérica.

8. La Red es interdependiente, en donde todos los investigadores y centros vinculados, comparten sus experiencias y promueven soluciones comunes.

9. La participación en la Red es netamente voluntaria, en donde se obvian los eventuales conflictos de interés únicamente individuales.

10. La Red obedece al principio de igualdad donde todos los participantes tienen los mismos derechos y obligaciones para con el o los proyectos y los pacientes con hipertensión pulmonar.

11. En la Red todos los procesos son abiertos y compartidos, con comunicación efectiva entre sus miembros.

12. La Red inicia como un compromiso formal entre profesionales de diversos centros nacionales que comparten el interés por la hipertensión pulmonar, que ha permitido dar el cuerpo general para la inclusión de otras sociedades, otros países de Latinoamérica, o personas de otros países inmersos en otras sociedades que a título personal, deseen pertenecer a ella.

13. Y por último, la Red está direccionada por objetivos, con el afán de ser facilitadora y operativa con productos específicos que beneficien la gestión de la enfermedad en la región y el resto de países de Latinoamérica.

\section{Personería jurídica de HAPredco}

La HAPredco aún no tiene personería jurídica en Colombia o fuera de ella. Sin embargo, uno de los requerimientos propios para la existencia de la Red es diligenciar la documentación y requisitos necesarios 
para disponer de dicha personería. En la actualidad, los aspectos presupuestarios y gestión de fondos se han centralizado en ASONEUMOCITO, tras haber obtenido su aval.

\section{Ventajas de la iniciativa HAPredco}

La verdadera riqueza de la Red se basa en que representa una organización de personas, desde los pacientes hasta los facultativos, incluyendo diferentes actores que exhiben diversas disciplinas de los sistemas de salud. La orientación de HAPredco tiene como valor intrínseco el trabajo común, gremial "en red", en donde (6):

- Lo común en red suma más que las acciones individuales (i.e., sinergia).

- El trabajo en red aporta legitimidad.

- El trabajo en red permite crear nodos de acción.

- El trabajo en red aumenta la capacidad de negociaciones.

- El trabajo en red facilita la cooperación en donde las necesidades de unos encuentran soluciones de otros.

- El trabajo en red aporta creación colectiva.

- El trabajo en red aporta mayor información.

- El trabajo en red ofrece mayores oportunidades.

- El trabajo en red aporta mayor motivación.

- El trabajo en red permite compartir logros.

\section{Instrumentos de la iniciativa HAPredco}

Los instrumentos de interacción continua lo representarán: la identificación de problemas específicos relacionados con la HP en Latinoamérica como principal insumo para la generación de preguntas de investigación y líneas de trabajo común; el registro de información de pacientes, la realización de actividades académicas, científicas o interacción específicas y viabilizar iniciativas e investigaciones propuestas por los propios miembros de la HAPredco. Estos instrumentos y el resultado de ellos, se expondrán en foros diversos definidos por la HAPredco, los establecidos por ASONEUMOCITO $\mathrm{u}$ otro consensuado entre los diversos actores. Todos los actores que intervienen en estos instrumentos y sus resultados, pueden hacer uso racional de ellos, en el marco normativo de ASONEUMOCITO y de cada uno de los establecidos por las sociedades integrantes, siempre con la autorización consensuada entre los líderes de cada centro.

\section{Amenazas potenciales para la HAPredco}

Todo trabajo en Red está expuesto a dos tipos de riesgo, uno relacionado con la producción y otro con la propia gestión. En el primero, existe posibilidad de tener más distracciones y pérdidas de tiempo, y que con ello disminuya el rendimiento comparado con acciones individuales. En el segundo, existe el riesgo de división en subgrupos y dificultades por criterios impositivos o maleficentes de una persona o de un grupo de personas. Pese a lo anterior, está demostrado que, a mayor cohesión y simbiosis de sus miembros, estos riesgos se disminuyen.

\section{Modelo de Gobernanza y Gestión de la HAPredco: Modelo de "Núcleo" y "Nodos" Operativos}

Nuestra propuesta ha definido que la Red cuente con un Núcleo o Centro Operativo, el cual estará encargado de centralizar, administrar y consolidar los aportes académicos y científicos de todos los miembros de la Red. Este núcleo o centro operativo será quien regule y administre todos los aportes académicos y científicos de todos los miembros de la Red, así como todos los instrumentos de interacción continua. Este núcleo difundirá, promoverá y gestionará las acciones de todos los Nodos o Centros Operativos Periféricos, los cuales estarán constituidos por cada centro participante independientemente del país o sociedad de origen. Esto permitirá que cada nodo o centro operativo periférico tenga la misma representatividad ante la Red, con la autonomía, voz y voto compartidos. De ésta forma, un país o sociedad podrá tener diversos y/o diferentes nodos o centros operativos periféricos, que estarán compartiendo una actividad u objetivo en común. Los miembros que constituyan éste núcleo o centro operativo tendrán vigencia limitada de acuerdo a la vigencia en el cargo, en la sociedad que representen. Una vez terminada la vigencia del cargo, estos miembros pasarán a ser consejeros de la Red, quienes además conservarán su voz y voto en el Núcleo o Centro Operativo, con lo que la operatividad se mantendrá de manera abierta. 


\section{Formas de participación en la HAPredco}

Nuestra iniciativa define tres formas fundamentales de participar en las acciones desarrolladas a través de La Red: Investigadores Principales, Co-investigadores y Miembros Aforados.

\section{Miembro Investigador Principal (MIP)}

La primera forma de participación es en calidad de MIP. Se define como aquel miembro que propone una iniciativa gremial ante una pregunta o grupo de preguntas delimitadas como un proyecto propio. Esta idea o ideas deberán ser registradas en el Núcleo o Centro Operativo, y se "embargará" en carácter de propiedad del investigador o investigadores principales. Proponemos que el embargo perdure por 6 a 12 meses, momento en el cual deberá existir un entregable (documento) tangible como artículo, resumen a congresos, grant u otro producto no definido en este documento. El entregable deberá consensuarse al inicio de la propuesta. Ante la aprobación de desarrollar la idea con datos comunes de la Red, el MIP deberá recibir los datos suficientes, pero de manera restringida para responder la pregunta de investigación. El MIP deberá aportar al menos tres informes breves del desarrollo del proyecto (al inicio, a los seis meses y al final) para difundirlos ante la Red. En caso de no aportar el entregable convenido en el tiempo definido, la idea o interrogante se desembargará y quedará de dominio público ante los miembros de la Red. Eventualmente, otro investigador principal podría desarrollarla bajo los mismos condicionantes. Para poder ser MIP se requiere tener al menos un paciente registrado en los instrumentos elaborados para tal fin y la validación del Núcleo o Centro Operativo.

\section{Miembro Investigador Colaborador (MIC)}

Es la segunda forma de participación, el MIC tendrá la oportunidad de participar activamente en la(s) iniciativas de un(os) MIP. Podrá ser MIP en caso de desembargo. Para ser MIC, solo se requiere de su experticia en un área específica del conocimiento que eventualmente esté relacionado a la hipertensión pulmonar y/o sirva a la red.

\section{Miembro Aforado (MA)}

La tercera forma de participación de la Red es en calidad de MA; se ha definido como el miembro del foro común de reuniones y acciones. Recibirá todas las informaciones perceptivas y las comunicaciones específicas de reuniones. Este aforado no aporta datos ni tampoco representa ser coautor de los entregables de la Red. A su vez, para poder ser considerado miembro aforado, solo requiere de su experticia en el área de la circulación pulmonar, hipertensión pulmonar o área afín, o que sirva en alguno de los requerimientos de la red. De las definiciones de MIP, MIC y MA, se desprende la necesidad de definir las coautorías principales y coautores principales. Sin embargo, todos los miembros debidamente inscritos en la Red gozarán de coautoría de los artículos y constarán bajo el epígrafe "En nombre de la HAPredco".

\section{Aspectos éticos e Información para la Base Cen- tral de Datos de HAPredco}

La información que se deposite en la base de datos central de la HAPredco es propiedad de los pacientes, quienes deberán aportar su consentimiento escrito para que sea gestionada bajo el presente modelo de gobernanza. La iniciativa de la HAPredco deberá contar con el aval del Comité de Ética de cada institución participante. Cada institución deberá tener como mínimo un investigador principal, quien será el interlocutor para los aspectos logísticos. No hay límite en cuanto al número de participantes por cada institución. Cada investigador o miembro deberá firmar el documento de confidencialidad y confluencia o conflicto de intereses (7).

Para digitar la información, la HAPredco cuenta con un gestor de la información que se encarga de la digitación de las variables disponibles (actualmente, casi 500 variables independientes) pero se ha creado un formato de captura que permite que cada investigador la digite si es de su preferencia.

El criterio sine qua non (excepto para casos de pediatría) para que un caso sea incluido en el análisis de la HAPredco, es la disponibilidad de un cateterismo pulmonar y cardíaco derecho que cumpla con la definición de hipertensión pulmonar y su clasificación en grupos 1, 2, 3, 4 o 5 . 


\section{Derechos y deberes de los miembros de la HAPredco}

\section{Iniciativas de investigación.}

Todo miembro de la HAPredco puede proponer una iniciativa de investigación en el área temática de la HP en un escrito formal y acompañado por la solicitud correspondiente. El autor(es) (MIP vs MIC) de esa propuesta tendrá el derecho de contar con la información de bases de datos propias de la HPredco. El autor o autores serán los encargados de definir(se) como el grupo ejecutivo que encabezará el trabajo y por ende la autoría principal. En todos los trabajos a publicar deberá llevar al final de los autores principales el epígrafe de "En nombre de la HPredco".

\section{Coautorías y consecución de Fondos.}

Para disponer del enunciado "En nombre de la HAPredco" que se refiere a la coautoría de la "Red", se deberá validar mediante la escritura de un documento y publicarlo, tomando en consideración las condiciones, modelos de gestión y gobernanza de la HPredco. La Red podrá generar documentos maestros de difusión gremial, así como mediante el consenso de ella, podrá emitir estrategias de colaboración con entes financiadores potenciales (p.ej., industria farmacéuti$\mathrm{ca}$, entes estatales u otras que defina la Red mediante consenso) o convenios con entidades internacionales académicas.

\section{Propiedad intelectual y patrimonial.}

La HAPredco propende porque las publicaciones en forma de resúmenes, congresos, artículos originales, y toda forma de material científico de difusión en reuniones gremiales (congresos, simposios) o académicas (universidades y similares) hagan constar a la red como entidad fuente original de información. La intelectualidad de las investigaciones propuestas y los productos obtenidos son propiedad de los autores, y se consensuará el orden desde los preliminares de las propuestas. Siempre se incluirá el epígrafe "en nombre de la Red Colombiana de Hipertensión Pulmonar" sucediendo al último autor. Las políticas de copyright y responsabilidades relacionadas con el contenido del artículo son adoptadas de las propias de ASONEUMOCITO.

\section{Declaraciones de Confluencia de Intereses y Compromiso de Confidencialidad}

El pertenecer a la Red en cualquiera de sus tres formas definidas (MIP, MIC y aforado), se realizará a través de la firma de dos documentos: en el primero cada miembro declarará su interés de pertenencia a la Red, su justificación para pertenecer a ella y la confluencia de intereses que pueda sesgar su independencia conceptual o de gestión. En el segundo, se propone la firma de un acuerdo de confidencialidad en el cual las comunicaciones e informaciones solo podrán hacerse tras previo acuerdo consensuado del Núcleo Operativo como representante del total de los miembros de la Red.

\section{Comité de arbitramento}

La HAPredco no prevé conflictos entre sus miembros. En caso de discrepancias respecto del funcionamiento, producción y referenciaciones de coautores, entre otros, se establece que el núcleo operativo ejercerá como comité de arbitramento para resolverlas siempre en beneficio de los integrantes y la propia red. El arbitramento podrá ser ampliado dependiendo de las necesidades de asesoría jurídica, legal, médica, ética o de otro tipo, según pueda requerirse en el caso eventual.

\section{Conclusiones}

La Red Colombiana de Hipertensión Pulmonar (HAPredco) fue creada bajo el concepto que su riqueza representa una organización de personas, con posibilidad de participación desde los pacientes hasta los facultativos de diversas disciplinas y especialidades del sistema de salud (8). La orientación de HAPredco concibe que el trabajo en red suma más que las acciones individuales, para la caracterización de los pacientes con HP en Colombia, así como la planificación de estrategias para resolver los problemas propios de su atención. Parte de los valores que justifican la iniciativa tienen que ver con que la red aporta legitimidad, permite crear nodos de acción, aumenta la capacidad de negociaciones, facilita la cooperación para que las necesidades de unos encuentren soluciones de otro; aporta creación colectiva, aporta mayor información, ofrece mayores oportunidades, aporta mayor motivación y, en definitiva, permite compartir logros. No podemos concluir sin hacer constar nuestra invitación a todas y todos quienes tengan intereses respecto de la HP para vincularse y aportar sus iniciativas a la HAPredco. 


\section{Referencias}

1. Kovacs G, Dumitrescu D, Barner A, Greiner S, Grünig E, Hager A, et al. Definition, clinical classification and initial diagnosis of pulmonary hypertension: Update recommendations from the Cologne Consensus Conference 2018. International Journey of Cardiology. 2018 Aug; 272(2018).

2. Galiê N, McLaughlin V, Rubin L, Simonneau G. An overview of the 6th World Symposium of Pulmonary Hypertension. Eur Respir J. 2019; 53(1802148).

3. Ficapal-Cusí P. La organización en red del trabajo: una aproximación empírica para la empesa catalana. uocpapers. 2008 abril; 8(2008).

4. Gregorietti V, Bortman G, Perrone S. Conformación de grupos de trabajo para estudio y tratamiento de la hipertensión arterial pulmonar. Inf Card. 2012 Oct; 7(4): p. 149-162.
5. Muijs D, West M, Ainscow M. ¿Por qué trabajar en redes? Aspectos teóricos. School Effectiveness and School Improvement. 2010; 21(1).

6. Gónzalez-Abad L, Rodríguez-Rodríguez A. El trabajo en red colaborativo: desafíos y posibilidades. Cuader. trab.soc. 2020; 33(1): p. 141-151.

7. McGoon M, Miller D. REVEAL: a contemporary US pulmonary arterial hypertension registry. Eur Respir Rev. 2012; 21(123): p. 8-18.

8. Paredes D. Trabajo en red, salud mental y calidad de vida para las personas con discapacidad intelectual y del desarrollo. International Journal of Developmental and Educational Psychology. 2014; 5(1): p. 371-376.

9. Barberá J, Román A, Gómez-Sánchez M, Blanco I, Otero R, López-Reyes R, et al. Guía de diagnóstico y tratamiento de la hipertensión pulmonar: resumen de recomendaciones. Arch Bronconeumol. 2018 febrero; 54(4): p. 205-215. 